\title{
Cognitive Deviation of TCM Caused by the Early Chinese Translation of Western Medicine*
}

\author{
Ya Liu \\ School of Foreign Languages \\ Hubei University of Chinese Medicine \\ Wuhan, China
}

\author{
Mingji Liu \\ School of Foreign Languages \\ Hubei University of Chinese Medicine \\ Wuhan, China
}

\author{
Yuhui Luo** \\ School of Foreign Languages \\ Hubei University of Chinese Medicine \\ Wuhan, China \\ **Corresponding Author
}

\begin{abstract}
In the early Chinese translation of western medicine, translators borrowed some terminologies from Traditional Chinese Medicine (TCM). Their inappropriate translation led to the confusion of terminological concepts and connotations of the two different theoretical systems. These loanwords resulted in people's misunderstanding towards TCM to some extent. Nowadays, in a world of western medicine's dominance, western medicine is regarded as the symbol of scientific medicine, and TCM is required to close up to the standard of it. However, the two different kinds of medicine differ largely in cultural connotations and theoretical systems, thus neither can be the standard of the other. The author holds that it's crucial to clarify the misunderstanding, to avoid the mistranslation, and to spread the cultural quintessence of the TCM to the world.
\end{abstract}

Keywords - translation of western medicine; TCM; deviation; influence

\section{INTRODUCTION}

Chinese People today seem to know more about western medicine, but hardly understand TCM. Western medicine, in which modern science are involved, is more understandable, objective, specific and acceptable. On the contrary, TCM tends to be more comprehensive, integral, abstract, interrelated, subjective and hard to understand, lacking of the so-called "elements of modern science". The focus of the cognitive deviation on the scientific evaluation of the two systems is the recognition of Chinese terminology on the same viscera organs and the explanation of the same disease. As western medicine predominates the modern medicine, it is considered as the standard to evaluate all the other medicines. In fact, TCM and western medicine are totally different medical theoretical systems with their distinctive advantages and characteristics in clinical application, thus

\footnotetext{
*Supported by 2017 China National Committee for Translation \& Interpreting Education Project (MTIJZW201708); Project of Humanities and Social Sciences of Ministry of Education of the People's Republic of China in 2018 (18YJA740031)
}

the two systems are totally incommensurable and neither can be the standard of the other. As the splendid traditional culture in China, TCM needs to be inherited and spread to the outside world. One of the most puzzling and difficult problems in the dissemination of TCM is language obstacles. To solve the problem and to correct people's cognitive deviation on TCM, we should trace to the source by reviewing the evolutionary history of TCM and western medicine, and studying the methods and the motivation of the early Chinese translation of western medicine.

\section{THE SPREAD OF WeStern MEDICINE INTO THE EAST IN EARLY MING AND QING DYNASTY}

TCM has a history of thousands of years, while the history of western medicine in China has only 400 years approximately. The time of the arrival of western medicine in China can be traced back to the Tang Dynasty. Whereas, the large-scale translation of western medicine started from the Late Ming and Early Qing Dynasty [1]. At that time, some western Christian missionaries who came to China began to engage in the translation of western medical works. When it came to the Late Qing and Early Min Dynasty, the translation activities changed into a whirlwind which swept through the whole country.

During that period, abundant of translated western medical works came out in succession: Dr. Joannes Terrenz, one of the missionaries, translated Taixi Ren Shuo Gai Lun and Ren Shen Tu Shuo [2], and he also translated Zhong Dou Qi Fa written by British doctor - Alexander Pearson. Afterwards, Benjamin Hobson and John Glasgou Kerr paved the way for the systematic translation of modern western medicine. Benjamin Hobson' $\mathrm{s}$ translation works can be listed as below: An Outline of Anatomy and Physiology, First Lines of the Practice of Surgery in the West, Practice of Medicine and Materia Medica, Treatise on Midwifery and Disease of Children, Medical Vocabulary and Medical Vocabulary in English and Chinese [3]. John Glasgou Kerr 
translated about 20 western medical works, namely Brief Introduction to Western Medicine, Brief Introduction to Ophthalmology etc. Additionally, British people John Hepburn, John Fryer - Dean of the Sao Paulo Academy in Hongkong, P. B. Cousland translated many works on medical theory, preclinical medicine, clinical treatment and pharmacy. According to the statistics, there were 111 translations of western medicine in all from 1899 to 1904.

Undoubtedly, western medicine in China located in inferior position in the Late Ming and Early Qing Dynasty, and it could not exist independently, let alone predominate the Chinese health cause. Before the Opium War, Chinese people were utterly ignorant of the new arrival of western medicine. The inferior status of western medicine was clearly known by these American missionaries who came to China and engaged in the introduction of western medicine. Therefore, they translated and published lots of western medical works to lay foundation for the spread of western medicine. In terms of translated works, these missionaries put forward some translation principles and methods, such as "free translation" which means searching for the vocabulary from the English, Chinese dictionaries. Because TCM has long been existed in China, naturally these translators chose the equivalent terms from TCM to translate terms in western medicine. Thus, it was easy for them to find the vocabulary concerning symptoms and zang-fu organs from TCM. Due to this translation method, western medicine was gradually accepted by the Chinese and rapidly spread to the whole nation.

\section{PeOPle's Cognitive DeVIATION ON TCM CAUSED BY THE EARLY CHINESE TRANSLATION OF WESTERN MEDICINE}

Except for these missionaries, some western doctors, botanists, pharmacologists who traveled around Asia in the 17 century also translated and spread western medicine consciously and systematically. Although they were not occupational or professional translators and they had never done any research on translation, they paved the way for the translation of TCM culture in western world without consciousness.

As to the translation methods, when spreading western medicine, these missionaries borrowed some TCM terms which had already existed thousands of years in China to make their translations acceptable for the Chinese. During this process, their misunderstanding of some TCM terms unavoidably led to their mistranslation of certain expressions, which also resulted in modern people's cognitive deviation of TCM and western medicine. The first deviation: owing to the effects of early Chinese translation of western medicine, people today have cognitive deviation on TCM, and they regard western medicine as the standard to evaluate the TCM. The second deviation: there exists deviation on the educational dissemination of TCM. Chinese medical culture was something does not accord with the western science, causing westerners create deviation on TCM culture. The misunderstanding or deviation can be seen as the results of the mistranslation or the missionaries' misunderstanding of TCM. The inappropriate loanwords caused the confusion of terminological concepts and connotations in the two different theoretical systems. In other words, TCM terms which were loaned by these translators misguided people's understanding of TCM.

As regards to the translation motivation, missionaries translated those western medical works for their preach activities and intended to make Chinese people accept western culture with the help of the carrier of western medicine. American Church Association in china claimed, "The best way to introduce Christianity to China is through western medicine..." [5] Accordingly, the attitude of devaluing and distorting TCM appeared in the early Chinese translation of western medicine. The preface of "An Outline of Anatomy and Physiology" also revealed the intention of the early translation of western medicine: "I have been to Guangdong for years. When I learn Chinese language in my spare time, I find the authors of TCM works know nothing about the exact positions of the bones, muscles, zang-fu organs, meridians and collaterals. They class the structure through experienced functions and do not care the concrete inner detail. I cannot help sighing because of their ignorant of this important knowledge. If they are not clear about the exact locations, how can they identify the root of disease accurately, and how can they cure the disease? ... Then western medical works can be adopted as reference. "[6] It's evident to find that missionaries at that time held a doubtful attitude towards TCM, so prejudices and misinterpretations of TCM existed in their translations. TCM and western medicine are totally different medical theoretical systems. To spread western medicine, these missionaries, who did not gain a deep insight into TCM, inappropriately borrowed TCM terms to translate the vocabulary in western medicine. Inescapably, some TCM terms and western medicine terms which have similar meanings were treated as the same. This caused the confusion of the two rivals. Although some organs with the same name have some similar functions in both TCM and western medicine, some of them differ largely in meaning. For example, Hobson translated the "heart" into "Xin", "kidney" into "Neishen" in his Medical Vocabulary in English and Chinese. As a matter of fact, the connotations of "Xin", "Shen" in TCM differ widely with "heart" and "kidney" in western medicine. The existence of "Xin" and "Shen" in TCM has a history of thousands of years, and they are not formed on the theory of anatomy and physiology in western medicine. By contrast, there are only hundreds of years of the history of "heart" and "kidney" in western medicine. Examples like this caused lots of troubles in people's understanding and recognition of TCM.

The inappropriate translation led western medicine to be the only scientific standard for people today, whereas TCM, from which TCM terms were borrowed by early translators, is required to accord with the standard of it. The disguised replacement of concepts led TCM to flow out in the Republic of China period. TCM was abandoned by the Northern Warlords Government in the reform of educational system in the first Reign year of the Republic of China, which ignited the first resistance and petition in the field of TCM. Since then, there were an increasing number of people who held the viewpoint of abolishing TCM. Yu Yunyou 
regarded TCM as a stumbling block in the way of the health cause in China, and he totally repudiated TCM by putting forward "The Act of Abolishing TCM to Remove the Barriers of Health Cause". Some scholars and politicians, including Yan Fu, Liang Qichao, Lu Xun, Guo Moruo, Chen Duxiu and Hu Shi, etc. [8], understood little about TCM and joined in the group of crusading against TCM. In consequence, the image and position of TCM was greatly damaged and weakened by the discrimination and attacks.

\section{THE DifFERENCES BETWEEN THE TwO KINDS OF MEDICAL THEORETICAL SYSTEMS}

The special culture and systematic theory of TCM differs widely with that of western medicine, and neither of the two paradigms can serve as a standard to inspect the other. The early Chinese translation of western medicine enabled the Chinese to know western medicine. Western medicine distinguishes itself by its clear aim, deep recognition and quick effect, so it is popularized easily. People today are accustomed to understanding and explaining TCM with the concepts and standards of western medicine. Actually, the medical theoretical systems of TCM and western medicine are totally unrelated with each other, thus people's cognitive deviation on TCM occurs. Westerners' prejudice and misunderstanding on TCM can also be blamed for the reason that the early translators of western medicine failed to understand and translate the theory and culture of TCM objectively and accurately. TCM, one of China's splendid cultural heritages, has a history of several thousands of years. It is a process dealing with human's recognition of phenomenon — symptoms — pathology — physiology. TCM summed up the experience of the Chinese people in their long struggle against diseases, and under the influence of ancient naive materialism, evolved into a unique, integral system of medical theory through long clinical practice. By analogy of classification, TCM holds that the functioning of our body interrelates closely to the natural laws of the world around us. And it also combines with unique culture of Chinese Confucianism, Taoism and Buddhism. By contrast, western medicine depends on the basic modern sciences, and scientific experiment is a basic tool in it. It's a process dealing with human' $s$ recognition of anatomy — tissues physiology - pathology. It regards each organ as an isolated part of human body, so it characterizes in its deep recognition of individual details but fails to treat human being as an organic whole and ignore the relation between human and nature.

The theoretical system of TCM includes many scientific elements which have not been explained by modern science yet. For example, TCM and western medicine have different recognition on human body' s structure : it seems that little difference lies in the external parts of human body and their functions between TCM and western medicine, but large difference can be found as to functions and interrelationships of internal viscera and organs. Take "Shen" as an example, TCM holds that the kidney is the origin of five viscera and, therefore, considered as the foundation of prenatal life. Its main physiological functions include the functions of urinary systems and reproductive system. The kidney also governs the bone, marrow, teeth and head hair. Meanwhile, the kidney's meridians connect with the urinary bladder, with which they are internally-externally related. However, western medicine holds that kidney is an organ which filters blood and generates urine. Evidently, the understanding of kidney in TCM is far from that in human anatomy of western medicine. The functions of organs in TCM are much more complex than that in western medicine. Evidences like this can be easily found in other viscera and organs.

\section{UNDERSTANDING OF INTER-TRANSLATION BETWEEN TCM AND WESTERN MEDICINE}

The way of borrowing words from other languages should be adjusted in the external transmission of TCM. If the translators of western medicine adopted the translation methods of transliteration plus free translation, perhaps the misunderstanding today can be avoided. Since terms like "heart" and "kidney" in western medicine have been equal with "Xin" and "Shen" in TCM, the bomb of disputes between TCM and western medicine can instantly be ignited. TCM terminologies, which have their specific meanings, have been used for thousands of years by Chinese people; however, with the invasion of western culture, western medicine, which distinguishes itself by its convenience, quick effect, and directness, was gradually accepted and rapidly spread in China. At the very beginning, western medicine was only a subsidiary of TCM, and then it developed to coexist with TCM, and then predominated the Chinese medical cause. Once it became the domination, it accused TCM concepts of being inaccurate, ambiguous and confusing. TCM terms are required to accord with the standard of western medicine, and this is the confusion caused by early Chinese translation of western medicine.

\section{CONCLUSION}

All in all, looking back on the developing history of Chinese translation of western medicine, we find that early missionaries borrowed TCM terms to spread western medicine which later predominates the Chinese medical cause and requires TCM to accord to its standard. However, the two theoretical systems are totally different, and inappropriate translation led to people's misunderstanding and cognitive deviation on TCM. It's impossible to change the history, but what we can do is to clarify and correct the misunderstanding; more importantly, we must avoid similar mistakes of spreading TCM in the future. In order to spread TCM to the outside world, "pin yin" with notes can be adopted to translate terminology and cultural elements in TCM. It is a better way to avoid misunderstanding and mistranslation, and to spread the source culture effectively. We should follow the principle of cultural equivalence and we can choose literal translation, free translation and transliteration properly to explain the cultural connotation of TCM and promote the development of TCM translation cause. 


\section{REFERENCES}

[1] Gao Liping, Missionary Translation and Modernity in the Culture and Society of the Late Qing Dynasty. Doctoral dissertation [D]. Shanghai: Shanghai International Studies University, 2011, pp. 75.

[2] Deng Tietao, Cheng Zhifan, A General History of Chinese Medicine (the Modern Volume) [M]. Beijing: People's Medical Publishing House, 2000, pp. 310.

[3] Chen Yongsheng, Zhang Sumeng, "Features of Translation of Western Medical Literatures and Publishing Institutions in Late Qing Dynasty ", National Medical Journal of China, vol. 2, pp. 76-81, Febuary 1997.

[4] Zhang Daqing, Gao Shilan, "A Promoter for the Standardization of the Medical Nomenclature ", China Historical Materials of Science and Technology, vol. 4, pp. 324-330, April 2001.

[5] Wang Jimin, “The Historical facts of Peter Parker's Invasion of China by Using Western Medicine ", Chinese Journal of Medical History, vol. 3, pp. 1, March 1951.

[6] Benjamin Terrenz, Chen Xiutang, An Outline of Anatomy and Physiology, The London Missionary Society Press, 1851, pp. 1-2.

[7] Source text of the decision of the central committee of public health on "the Act of Abolishing TCM. Medical Spring and Autumn Community, vol. 34, pp. 9.

[8] Zhao Hongjun, The Debating History on TCM and Western Medicine, Beijing: Academic Publishing House, 2012, pp. 21. 